\title{
LLENADO DE UN RECIPIENTE: MODELACIÓN Y SIMULACIÓN DE CONCEPTOS BÁSICOS AFINES A LA INGENIERÍA
}

FILLING A CONTAINER: MODELING AND SIMULATION OF BASIC CONCEPTS RELATED TO ENGINEERING

\author{
Luz Elena Tinoco Robledo* \\ https://orcid.org/0000-0001-5250-3839 \\ UNIVERSIDAD DE AMÉRICA, BOGOTÁ, COLOMBIA
}

Recibido: 11 de diciembre de 2019

Aceptado: 24 de enero de 2020

DOI: https://doi.org/10.29097/2011-639X.277

\section{Resumen}

Se caracteriza el comportamiento de llenado de un recipiente con dos fluidos: miel y lubricante. Inicialmente, se describen de forma cualitativa las variables y se procede a la experimentación registrando las mediciones. Posteriormente se realiza el análisis de datos, caracterizando de las variables para definir las relaciones entre estas, y se procede a la construcción de un modelo ajustado a los resultados obtenidos. Desde la experimentación, y según el modelo asociado a esta, se construye la simulación del evento a través de Geogebra, y se repite el proceso con cada una de las transformaciones del evento, permitiendo su análisis y comparación. Los resultados podrán ser usados como herramienta educativa idónea para la integración de áreas del conocimiento afines a la ingeniería.

Palabras clave: ciencias exactas, ciencias naturales, ingeniería, razonamiento, simulación

\section{Abstract}

The behavior of a container filled with two fluids, honey and lubricant, is characterized. Initially, we will describe the variables qualitatively and proceed to experimentation recording the measurements. Subsequently, the data analysis is performed and through the characterization of the variables the relationships between the parameters are determined, and a model adjusted to the results obtained is constructed. From the experimentation, and according to the model associated with it, the simulation of the event is constructed through Geogebra, and the process is repeated with each of the transformations of the event for its analysis and comparison. The results can be used as an ideal educational tool for the integration of areas of basic knowledge related to engineering.

Keywords: exact sciences, natural sciences, engineering, reasoning, simulation.

\footnotetext{
* Licenciada en matemáticas, Universidad Distrital Francisco José de Caldas. Maestrante en Enseñanza de Ciencias Exactas y Naturales, Universidad Nacional de Colombia. Docente Ciencias y Humanidades, Fundación Universidad de América. 凶 $\underline{\text { luz.tinoco@profesores.uamerica.edu.co }}$
} 


\section{INTRODUCCIÓN}

Dentro de los programas de ingeniería que oferta la Facultad de Ingeniería de la Fundación Universidad de América se encuentran algunos cursos transversales de la Unidad de Ciencias Básicas, pertenecientes al ciclo básico de formación, siendo estos pilares del conocimiento ingenieril, tal como lo enuncia el artículo 1 de la Ley 842 del 2003, a partir de la siguiente definición de ingeniería: "Se entiende por ingeniería toda aplicación de las ciencias físicas, químicas y matemáticas, de la técnica industrial y en general del ingenio humano a la utilización e invención sobre la materia”. Por otro lado, Acofi (2007, p. 14) presenta al ingeniero como alguien que "da fe de que sabe hacer obras particulares que resuelven necesidades de primer orden y cuya utilidad se afinca en el ejercicio de unos usos técnicos especializados”.

Esta definición particular nos permite reconocer la importancia de las ciencias dentro del ejercicio profesional de un ingeniero, en la medida en que, según Triana (2012, p. 51), el conocimiento asociado a las ciencias exactas y naturales "otorga al ingeniero la capacidad para otear y dimensionar las relaciones y estructuras entre objetos, y entidades, para poder entonces construir una sólida base de acción tecnológica funcional a partir de su aprendizaje significativo", esto desde tres ciencias básicas: las matemáticas, que desarrollan el pensamiento crítico y permiten la rigurosidad del mismo; la física, que permite la explicación de los fenómenos dada su naturaleza; y la química, desde la caracterización de la materia.

Por esta razón, se pretende abordar algunos de los contenidos pertenecientes a las ciencias descritas desde la aplicación de las mismas a un problema particular: el llenado de un recipiente con fluidos de propiedades distintas, que impliquen el desarrollo del razonamiento covariacional mediante la caracterización de fenómenos reales, y la posterior simulación en un software libre de reconocimiento mundial asociado a geometría dinámica, siendo esta una actividad investigativa que puede desencadenar posteriormente en la utilización de resultados y del software dentro de los procesos de enseñanza-aprendizaje, desarrollando practicas significativas y guiadas por la experiencia obtenida.

La situación problema que se pretende abordar se asocia con la labor del docenteinvestigador, en función de las actividades que pueden proponerse posteriormente a partir de los resultados obtenidos, por lo que se revisan algunos referentes teóricos que no solo aportan a las intenciones del experimento inicial, sino que también pueden relacionarse con su utilidad en el contexto educativo.

En algunas investigaciones, como las de Johnson (2015) y Paoletti \& Moore (2017), se les pregunta a los estudiantes por el cambio del volumen de agua con respecto a la altura de llenado de botellas de diferentes formas, pidiendo que lo representen en una gráfica. A pesar de que esta relación es fácilmente reconocible, varios estudiantes han demostrado algunas dificultades en la solución de dicha situación. Lo interesante del tema es cómo a partir del análisis de variables paramétrizadas en función del tiempo se define matemáticamente el comportamiento de la tasa de llenado o de vaciado con respecto al volumen y/o a la altura, tanto a tasas constantes como a tasas variables; permitiendo la involucración del estudiante en contextos covariacionales. 
Así, se establece que a raíz de este ejercicio un estudiante conceptualiza dos cantidades que varían con respecto al tiempo conceptual -el tiempo conceptual es definido como una imagen de la medida de la duración, donde a diferencia del tiempo experimental no se cronometra un evento sino que se tiene una idea de cómo una cantidad tiene diferentes valores en diferentes momentos previendo una ocurrencia de forma continua y con variación constante- representa y coordina esas dos cantidades, de forma tal que una variación en cualquiera de las dos necesariamente implica variación en la otra cantidad (Moore \& Thompson, 2015).

En función de este problema, Carlson et al. (2002) han descrito lo que se conoce como acciones mentales (MA) en relación al problema de la botella, pues cuando se coordina la relación entre el volumen y la altura del agua mientras que esta es vertida dentro de la botella, el estudiante primero concibe la idea de que las dos magnitudes están cambiando (MA 1), para determinar luego que como el volumen de agua incrementa, la altura también incrementa (MA 2). Posteriormente, el estudiante puede conceptualizar que, para cambios iguales en el volumen, representados por cada sección transversal coloreada en la figura 1. Los incrementos sucesivos en la altura disminuyen hasta la parte más ancha de la botella, después de lo cual la altura aumenta hasta el cuello de la botella (MA 3). Cuando el estudiante reconstruye esta relación, puede determinar la tasa promedio (MA 4) e instantánea (MA 5) de cambio de volumen de líquido con respecto a la altura una vez el agua es vertida dentro de la botella.

\section{Figura 1.}

Representación del llenado de un recipiente cilíndrico.

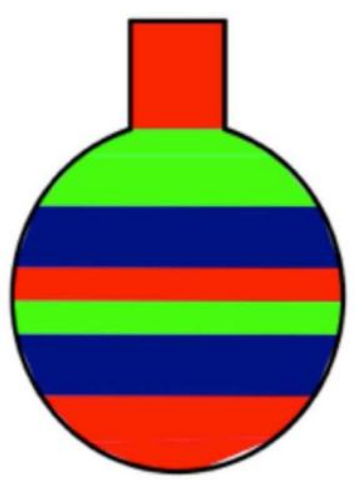

Nota. Se observa el volumen ocupado por un líquido en un recipiente con caudal constante, las particiones hacen referencia a un mismo intervalo de tiempo de llenado. Fuente: Paoletti (2017, p. 141).

Desde otras investigaciones, la covariación puede ser definida como estática, cuando los valores de una cantidad están asociados con valores de alguna otra cantidad, o dinámica, cuando el cambio en una cantidad está asociado con cambios en otra cantidad (Clement, 1989). Con respecto a la covariación estática, un ejemplo es cuando se hace variar la longitud del lado de un cuadrado de $2 \mathrm{~cm}$ a $3 \mathrm{~cm}$, y por ende el área del cuadrado 
cambia de $4 \mathrm{~cm}^{2}$ a $9 \mathrm{~cm}^{2}$. Por otra parte, cuando se quiere ejemplificar la covariación dinámica es posible tener dos escenarios: discreto y continuo. Una covariación dinámica discreta involucra la correlación del cambio entre valores sucesivos en una cantidad con el cambio entre valores asociados a la otra cantidad; es decir, siguiendo el ejemplo anterior, se relaciona la variación de la longitud $(1 \mathrm{~cm})$ con respecto a la variación en área $\left(5 \mathrm{~cm}^{2}\right)$. En cuanto a la covariación dinámica continua, esta implica la correlación del cambio continuo de una cantidad con el cambio continuo en otra cantidad, según Saldanha \& Thompson (1998). Por ejemplo, cuando la longitud del lado del cuadrado incrementa continuamente, se tiene que su área incrementa también a tasa constante.

En cuanto al razonamiento transformacional, Simon (1996) desarrolló investigaciones con Geometric Supposer Software para establecer relaciones entre los ángulos de la base y los lados de un triángulo isósceles. Para nuestra investigación se hizo uso de este tipo de razonamiento mediante un software de matemática dinámica llamado GeoGebra, promoviendo el razonamiento covariacional a través de la emulación de las variables que interactúan en el fenómeno y la proyección geométrica del mismo. Básicamente, la meta es lograr que un individuo encuentre el sentido y la función de las matemáticas al realizar el razonamiento transformacional.

\section{RAZONAMIENTO COVARIACIONAL}

Para Johnson (2013) y Stalvey \& Vidakovic (2015), la covariación se refiere a un fenómeno matemático donde dos o más cantidades cambian simultánea e interdependientemente en un sistema dinámico; también se ha establecido que la noción de covariación es "la de alguien que tiene en mente una imagen sostenida de los valores (magnitudes) de dos cantidades simultáneamente (Saldanha \& Thompson, 1998, p. 1). Esto implica que se pueden matematizar diferentes fenómenos de la realidad en función y relación de varias dimensiones y cantidades, las cuales permitan al estudiante aprehender un conocimiento a partir de la experiencia y la teoría. La covariación puede ser establecida en dos formas diferentes:

- La correlación de dos cantidades como si estas estuviesen cambiando independientemente una de la otra, denominado razonamiento independientesimultáneo (Thompson, 2011); y

- El razonamiento de cómo una cantidad varía en respuesta a los cambios de la otra, denominado razonamiento cambio-dependiente (Johnson, 2012). También implica una experiencia mental donde "se coordinan dos cantidades variables mientras que se atiende de las formas en que cambian entre sí” (Carlson et al., 2002).

\section{METODOLOGÍA}

La metodología de esta investigación está construida a partir de los objetivos propuestos, los cuales son: 
- Analizar las evidencias existentes con respecto al desarrollo del pensamiento covariacional desde la situación del llenado de una botella y las variaciones de este fenómeno.

- Recolectar datos propios del análisis cualitativo y cuantitativo a partir de la observación, la experimentación y la comprobación, permitiendo la caracterización de un modelo inicial básico que describa el llenado de una botella y sus variaciones.

- Analizar los datos obtenidos desde la experimentación, construyendo los modelos asociados a cada situación, junto con las consideraciones teóricas que explican el comportamiento del fluido en cada una de ellas.

- Usar las habilidades obtenidas en el análisis y modelación de los eventos físicos para la representación y simulación del llenado de una botella y sus variaciones en Geogebra (software interactivo predeterminado de geometría dinámica).

- Comparar los resultados obtenidos desde las simulaciones del fenómeno con los datos obtenidos del mismo a través de la experimentación.

- Integrar los eventos de simulación en un único archivo de Geogebra que permita la comparación en tiempo real de los eventos realizados.

Para lograr los objetivos descritos se plantea la siguiente metodología:

- En principio se analizaron las evidencias existentes con respecto al desarrollo del pensamiento covariacional desde la situación de llenado de una botella y las variaciones de este fenómeno. Para ello se hizo la recolección de información y literatura pertinente para el entendimiento, ejecución, puesta en marcha, desarrollo de estrategias, conclusiones, problemas y retos que se proponen alrededor del pensamiento covariacional para la resolución de problemas en ingeniería.

- Se realizó el diseño de la situación problema general, referida al llenado de una botella cilíndrica — lo cual cae en el campo de la mecánica de fluidos—, fijando condiciones de operación y estableciendo las variables que se pretenden correlacionar para la descripción del fenómeno desde el punto de vista físico (temperatura, caudal), químico (viscosidad, composición) y matemático (altura de la columna, relación área-volumen), en la medida en que puedan alterar el comportamiento del mismo.

- Se generaron hipótesis de trabajo alrededor de las diferentes configuraciones, para ser verificadas posteriormente a partir de la experimentación.

- Se desarrolló la propuesta de diseño de experimento y su ejecución, haciendo la respectiva recolección, tabulación y comparación de los datos obtenidos experimentalmente.

- Se analizaron los datos obtenidos previamente a partir de la observación y la evidencia de la experimentación, permitiendo la caracterización de un modelo básico que describe el llenado de una botella y sus variaciones. 
- Se generaron posibles distribuciones y/o perfiles de las variables involucradas en el fenómeno, y se redactaron los análisis de resultados y las conclusiones en relación con las hipótesis planteadas.

- A partir de los modelos obtenidos para cada una de las situaciones se construyó la simulación en el software libre Geogebra, considerando las características del envase, las variables definidas y los resultados obtenidos.

- Finalmente, se establecieron comparaciones entre los resultados de las simulaciones de los modelos propuestos y los datos obtenidos del fenómeno real a través de la experimentación, revisando también la concordancia entre los mismos, con la finalidad de evidenciar la relación teórico-experimental.

\section{DESCRIPCIÓN DE LA EXPERIMENTACIÓN}

Tanto para la situación general como para las variaciones, la experimentación se desarrolló colocando fluido en el viscosímetro Saybolt, y procediendo a retirar el corcho del envase que lo contiene para dejar caer el líquido sobre un recipiente, el cual, para los eventos trabajados en la investigación, se reduce a una probeta de forma cilíndrica. Los pasos de la experimentación se exponen a continuación:

- Definición de fluidos de trabajo: lubricante de transmisión SAE10W90 y miel (tipo syrup).

- Establecimiento de celda de trabajo: Celda Furol. Los fluidos a temperatura ambiente presentan viscosidades mayores a 1000 S.S.F (Segundos Saybolt Furol).

- Definición de la columna de fluido en el recipiente que se vacía: $60 \mathrm{~mL}$, según medidas estándar de la norma ASTM D-88.

- Definición del recipiente de llenado: probeta cilíndrica de $40 \mathrm{~mL}$.

- Tarado del recipiente en cm: cinta de $12 \mathrm{~cm}$ y de $24 \mathrm{~cm}$ para el último evento.

- Definición de la temperatura: Para los primeros 8 eventos la temperatura del fluido desalojado permanece constante, a excepción del evento 9 (donde la temperatura aumenta con el paso del tiempo).

- Toma de tiempos de caída: cronometrando cada centímetro de altura. Trazado de gráficas: recopilación de datos llevados a Excel.

- Realización de análisis pertinentes.

En total se realizaron dos eventos iniciales y siete variaciones de ellos, para un total de nueve eventos que se describen a continuación:

Evento 1: Lubricante de transmisión a una temperatura de $20{ }^{\circ} \mathrm{C}$ con columna variable (lo que significa que se vacía la celda, perdiendo altura conforme avanza el tiempo).

Evento 2: Miel a una temperatura de $30^{\circ} \mathrm{C}$ con columna variable. 
Del primer evento, constituido por la experimentación con lubricante de transmisión SAE $10 \mathrm{~W} 90$ a una temperatura constante de $20^{\circ} \mathrm{C}$ con columna variable, se proceden a considerar los siguientes tres eventos:

Evento 3: Lubricante a una temperatura de $40{ }^{\circ} \mathrm{C}$ con columna variable.

Evento 4: Lubricante a una temperatura constante de $40{ }^{\circ} \mathrm{C}$ con columna de fluido constante.

Evento 5: Lubricante a una temperatura constante de $60{ }^{\circ} \mathrm{C}$ con columna de fluido constante.

Del segundo evento, constituido por la experimentación con miel a una temperatura constante de $20{ }^{\circ} \mathrm{C}$ con una columna de fluido variable, se proceden a considerar los siguientes cuatro eventos:

Evento 6: Miel a una temperatura de $60^{\circ} \mathrm{C}$ con columna variable.

Evento 7: Miel a una temperatura constante de $40{ }^{\circ} \mathrm{C}$ con columna de fluido constante.

Evento 8: Miel a una temperatura constante de $60{ }^{\circ} \mathrm{C}$ con columna de fluido constante.

Evento 9: Miel a una temperatura variable desde los $20^{\circ} \mathrm{C}$ hasta los $60{ }^{\circ} \mathrm{C}$ con columna de fluido variable.

\section{FORMULACIÓN DE HIPÓTESIS}

De acuerdo con los eventos se consideraron las siguientes hipótesis:

H (1): Cuando la columna del fluido es variable (mientras transcurre el tiempo el líquido en la celda se está vaciando y pierde altura), el caudal y la velocidad de llenado del recipiente disminuyen conforme avanza el tiempo, definiendo entonces una recta con pendiente negativa que describe el comportamiento de la velocidad de llenado en función del tiempo.

H (2): Cuando la columna del fluido es constante (mientras transcurre el tiempo la altura del líquido en el viscosímetro no cambia), el caudal y la velocidad de llenado del recipiente es constante.

H (3): El comportamiento de la altura depende de la columna del fluido: si esta última es constante la altura en el recipiente aumentará proporcionalmente con respecto al tiempo; por el contrario, si la columna es variable el cambio de la altura en el recipiente con respecto al tiempo (velocidad de llenado) definirá una recta con pendiente negativa. 
H (4): Para los eventos en los que la temperatura permanece constante, a altas temperaturas el desalojo del fluido en la celda se da a mayor velocidad. Además, para los casos en los que la altura de la celda es variable (Disminuye con el paso del tiempo) la variación en el caudal que irá en disminución por el principio de Torricelli será menos evidente. Esto significa que un evento a alta temperatura definirá un caudal en función del tiempo como una recta de pendiente negativa declinada si se compara con otro evento en las mismas condiciones (columna variable y temperatura constante), pero con temperatura de menor magnitud.

\section{RESULTADOS}

Con cada uno de los eventos mencionados se registraron datos asociados entre alturas predefinidas de la probeta usada y el tiempo transcurrido para que el fluido alcance cada una de ellas. Haciendo uso de herramientas multimedia se calcula el volumen para cada uno de los tiempos dados, consiguiendo así un modelo para la velocidad de llenado (cambio de la altura con respecto al tiempo) y un modelo para el caudal (cambio de volumen respecto al tiempo).

Teniendo en cuenta la experimentación y el análisis de cada uno de los eventos, se presenta una síntesis que agrupa los eventos iniciales y las variaciones que se dieron a estos. Cabe resaltar que las variables que se modificaron en cada uno de los eventos estuvieron asociadas al tipo de fluido, a la variación o no de la columna del fluido en la celda Furol, a la temperatura a la que se expuso el fluido y, en el último caso (evento 9), a la variación de la temperatura dentro de un mismo evento.

Para representar cada uno de los modelos encontrados para las variables relacionadas en función del tiempo, se presentan en la tabla 1 las consideraciones de cada uno de los eventos y los modelos construidos para relacionar tanto la altura con respecto al tiempo como la velocidad respecto al tiempo. También se presenta el tiempo total para alcanzar una altura de $12 \mathrm{~cm}$ en una probeta de radio igual a $1 \mathrm{~cm}$ (para el evento 9 se debe hacer una consideración adicional, al utilizar una altura doble de la inicial).

Por último, para cada uno de los eventos se encuentra el link de la simulación en el software Geogebra, en el que se evidencia el elemento con el que se realizó la experimentación, la réplica de la observación en el contexto real, y el comportamiento gráfico de la altura en función del tiempo.

\section{DATOS GENERALES}

- Volumen total en celda y en probeta: $34,02 \mathrm{~cm}^{3}$ (para el evento 9: 70,88 $\mathrm{cm}^{3}$ ).

- Radio de la celda: $1,35 \mathrm{~cm}$.

- Radio del orificio de la celda: $0,9 \mathrm{~cm}$.

- Radio de la probeta: $1 \mathrm{~cm}$.

- Altura alcanzada en la probeta: $12 \mathrm{~cm}$ (para el evento 9: $24 \mathrm{~cm}$ ). 


\section{Tabla 1.}

Eventos, modelos considerados y simulación

\begin{tabular}{|c|c|c|c|c|c|c|c|}
\hline \# Exp & Fluido & Temperatura & Columna & Altura vs. tiempo & Velocidad vs. tiempo & $\begin{array}{l}\text { Tiempo } \\
\text { total (s) }\end{array}$ & Link \\
\hline 1 & $\begin{array}{l}\text { Lubricante de } \\
\text { transmisión } \\
\text { SAE 10W90 }\end{array}$ & $\begin{array}{l}\text { Constante } \\
20^{\circ} \mathrm{C}\end{array}$ & Variable & $A(t)=-0,0002 t^{2}+0,1041 t$ & $V(t)=-0,0004 t+0,1089$ & $165,9 \mathrm{~s}$ & $\underline{1}$ \\
\hline 2 & Miel & $\begin{array}{l}\text { Constante } \\
30^{\circ} \mathrm{C} \\
\end{array}$ & Variable & $A(t)=-0,0003 t^{2}+0,03960 t+0,11794$ & $V(t)=-0,006 t+0,0396$ & $428,84 \mathrm{~s}$ & $\underline{2}$ \\
\hline 3 & $\begin{array}{l}\text { Lubricante de } \\
\text { transmisión } \\
\text { SAE 10W90 }\end{array}$ & $\begin{array}{l}\text { Constante } \\
40^{\circ} \mathrm{C}\end{array}$ & Variable & $A(t)=-0,0015 t^{2}+0,2797 t+0,1406$ & $\begin{array}{c}V(t)=-0,00002 t^{2}+0,00293 t \\
+0,31411\end{array}$ & $62,05 \mathrm{~s}$ & $\underline{3}$ \\
\hline 4 & $\begin{array}{l}\text { Lubricante de } \\
\text { transmisión } \\
\text { SAE 10W90 }\end{array}$ & $\begin{array}{l}\text { Constante } \\
40^{\circ} \mathrm{C}\end{array}$ & Constante & $A(t)=0,3633 t+0,002$ & $V(t)=0,364$ & $33 \mathrm{~s}$ & $\underline{4}$ \\
\hline 5 & $\begin{array}{l}\text { Lubricante de } \\
\text { transmisión } \\
\text { SAE 10W90 }\end{array}$ & $\begin{array}{l}\text { Constante } \\
60^{\circ} \mathrm{C}\end{array}$ & Constante & $A(t)=0,8302 t+0,0125$ & $V(t)=0,8302$ & $14,4 \mathrm{~s}$ & $\underline{5}$ \\
\hline 6 & Miel & $\begin{array}{l}\text { Constante } \\
60^{\circ} \mathrm{C}\end{array}$ & Variable & $A(t)=-0,0001 t^{2}+0,07305 t+0,0811$ & $V(t)=-0,0002 t+0,0765$ & $236,66 \mathrm{~s}$ & $\underline{6}$ \\
\hline 7 & Miel & $\begin{array}{l}\text { Constante } \\
40^{\circ} \mathrm{C}\end{array}$ & Constante & $A(t)=0,0552 t+0,0054$ & $V(t)=0,0552$ & $216,82 \mathrm{~s}$ & $\underline{7}$ \\
\hline 8 & Miel & $\begin{array}{l}\text { Constante } \\
60^{\circ} \mathrm{C}\end{array}$ & Constante & $A(t)=0,29146 t+0,00003$ & $V(t)=0,29146$ & $41,18 \mathrm{~s}$ & $\underline{8}$ \\
\hline 9 & Miel & $\begin{array}{c}\text { Variable } \\
\text { creciente } \\
20^{\circ} \mathrm{C}-60^{\circ} \mathrm{C}\end{array}$ & Variable & $A(t)=0,00003 t^{2}-0,00457 t+0,40785$ & $V(t)=0,00003 t-0,002$ & $677,38 \mathrm{~s}$ & $\underline{9}$ \\
\hline
\end{tabular}

Nota. Link de compilación de los 9 eventos: https://www.geogebra.org/classic/bzhhgpaw.

Fuente: Elaboración propia. 


\section{COMPARACIÓN DE HIPÓTESIS DE ACUERDO CON LOS RESULTADOS}

A continuación, se exponen cada una de las hipótesis propuestas, teniendo en cuenta los dos eventos iniciales y sus modificaciones a partir de la comparación entre las observaciones desde la experimentación, la recolección de los datos y el posterior análisis de lo sucedido en cada una de las situaciones descritas.

H (1): La hipótesis fue cierta para los eventos 1, 2, 3 y 6, donde la temperatura permaneció constante con respecto al paso del tiempo. Cuando la columna es variable, es decir cuando la altura del recipiente vaciado disminuye con respecto al tiempo, la velocidad de llenado y el caudal se ven afectados, pues la altura del recipiente de llenado y el volumen aumentan, pero este aumento es cada vez menor, luego la velocidad y el caudal están descritos por un modelo lineal decreciente en términos del tiempo. Si esto se define por un modelo lineal decreciente, el volumen aumentará, pero a menor altura de columna más tiempo tardará en vaciarse el fluido, por lo que su variación será cada vez menor. Cabe resaltar que para el evento 9 la hipótesis no aplica, pues allí se tiene una columna variable pero la temperatura aumenta a su vez, lo que conduce a tener un comportamiento distinto en la medida en que la variabilidad de la columna conduce a ralentizar la velocidad de llenado, pero el aumento de temperatura acelera la misma.

H (2): La hipótesis fue válida para los casos que cumplían las condiciones, es decir los eventos 4, 5, 7 y 8. Cuando la columna del fluido en la celda Furol se mantuvo constante la velocidad de llenado, y por ende, el caudal, describieron un comportamiento constante a través del tiempo, lo que llevó a que el aumento del volumen estuviera definido por un modelo lineal cuya pendiente es la constante mencionada (caudal).

H (3): La hipótesis fue válida en las experimentaciones propuestas, generaliza las hipótesis previas y permite la comparación de los eventos 1, 2, 3 y 6 (columna variable) con respecto a los eventos 4, 5, 7 y 8 (columna fija). En los eventos descritos a temperatura constante se evidencia que cuando la columna de fluido es constante la altura es proporcional al tiempo, por lo que el modelo se asocia siempre a un modelo lineal creciente de velocidad constante; para los eventos en los que la columna del fluido disminuye, la altura del recipiente de llenado tiene un aumento no uniforme, es decir la velocidad de llenado va siendo menor con respecto al tiempo. En esta última afirmación cabe resaltar que la velocidad de llenado en función del tiempo no siempre se definió por un modelo lineal decreciente, porque el evento 2 se definió bajo un modelo cuadrático local, en el que también se evidenció la disminución de la velocidad con respecto al paso del tiempo.

H (4): La hipótesis es válida, y puede corroborarse a partir de los tiempos calculados en los que cada evento experimental alcanzó la altura máxima propuesta en el recipiente. Para demostrar lo descrito en la hipótesis se compararán algunos eventos en los que se modificó únicamente la temperatura, para evidenciar particularmente este cambio desde el tiempo transcurrido: 
- En los eventos 1 y 3 se trabaja el mismo fluido (lubricante) con columna variable, pero el evento 1 se hace a una temperatura de $20^{\circ} \mathrm{C}$ con un tiempo de 165,9 s, mientras que el evento 3 se hace a una temperatura de $40{ }^{\circ} \mathrm{C}$ con un tiempo de $62,05 \mathrm{~s}$.

- En los eventos 4 y 5 se trabaja con lubricante y columna constante. En el evento 4 , con una temperatura de $40{ }^{\circ} \mathrm{C}$ se alcanza la altura máxima en $33 \mathrm{~s}$, mientras que en el evento 5 , a una temperatura de $60^{\circ} \mathrm{C}$ el fluido alcanza la altura máxima en 14,4 s, tiempo menor que todos los anteriores eventos, dado que tiene la mayor temperatura y a la vez la columna es constante.

- En el caso de la miel, en la comparación de los eventos 2 y 6, ambos con columna variable, el evento 2 tardó $428,84 \mathrm{~s}$ a una temperatura de $30^{\circ} \mathrm{C}$, mientras que el evento 6 tardó apenas 236,66 s a una temperatura de 40 ${ }^{\circ} \mathrm{C}$.

- En los eventos 7 y 8 con columna constante, la miel a $40^{\circ} \mathrm{C}$ tardó 216,82 $\mathrm{s}$ en completar la altura en el recipiente, mientras que a $60^{\circ} \mathrm{C}$ lo hizo en $41,8 \mathrm{~s}$.

Teniendo en cuenta el desarrollo de H (1), se evidencia una caracterización del llenado de la probeta dependiendo de la caracterización del vaciado de la celda del viscosímetro Saybolt, debido a que se aplica el principio de Torricelli, que determina el flujo de un líquido contenido en un recipiente que sale a través de un orificio.

\section{MODELO TEÓRICO PARA COLUMNA VARIABLE}

Según lo analizado en cada uno de los eventos donde se trabajó con columna variable (1, 2, 3 y 6), se buscó un modelo que permita definir el comportamiento de la altura de la celda mientras se está vaciando.

Tenemos inicialmente, por el principio de conservación de energía mecánica:

$$
E c=E p
$$

Luego

$$
\begin{gathered}
\frac{1}{2} m v^{2}=m g h \\
\frac{1}{2} v^{2}=g h \\
v^{2}=2 g h
\end{gathered}
$$




$$
v=\sqrt{2 g h} \quad \text { (ecuación } 1)
$$

Donde $g$ = aceleración de la gravedad, $h=$ altura del recipiente y $v=$ velocidad.

Según el principio de Torricelli, el caudal $\frac{d V}{d t}$ que fluye por un agujero se puede medir como el producto del área del orificio de salida por la velocidad del fluido, así:

$$
\frac{d V}{d t}=-a \cdot v \quad(\text { ecuación 2) }
$$

En condiciones reales, como lo expuesto en nuestra investigación se requiere considerar una constante $k$, equivalente al coeficiente de descarga, el cual depende de las características del fluido. Este factor es adimensional, teniendo que un mayor coeficiente de descarga implica mayor caudal, lo que significa que el fluido desembocará más rápido. Su valor está entre 0 y 1 . Así:

$$
\frac{d V}{d t}=-a \cdot k \cdot v \quad(\text { ecuación 3) }
$$

Donde $V=$ volumen, $t=$ tiempo, $a$ = área del orificio de salida.

Sustituyendo la ecuación 1 en la ecuación 3 se obtiene:

$$
\frac{d V}{d t}=-a k \sqrt{2 g h} \quad \text { (ecuación 4) }
$$

Por otro lado, sabemos que podemos obtener el cálculo del volumen por secciones transversales, teniendo en cuenta el área de la sección transversal $A(h)$ a una altura $h$ :

$$
V=\int_{0}^{h} A(h) \cdot d h
$$

Esta expresión se deriva con respecto al tiempo, obteniendo así lo que hemos denominado caudal expresado en términos del producto del área transversal por el cambio de la altura con respecto al tiempo.

$$
\frac{d V}{d t}=A(h) \frac{d h}{d t} \quad \text { (ecuación 5) }
$$


Igualando las ecuaciones 4 y 5, se obtiene:

$$
A(h) \frac{d h}{d t}=-a k \sqrt{2 g h}
$$

Teniendo en cuenta que $A(h)=\pi R^{2}$ (al ser un envase cilíndrico el área de la sección transversal es constante) y $a=\pi r^{2}$, donde $R=$ radio del recipiente y $r=$ radio del orificio de salida, se obtiene:

$$
\pi R^{2} \frac{d h}{d t}=-\pi r^{2} k \sqrt{2 g h}
$$

Luego, separando variables:

$$
\begin{aligned}
& R^{2} \frac{d h}{d t}=-r^{2} k \sqrt{2 g} \sqrt{h} \\
& \frac{1}{\sqrt{h}} d h=-\frac{r^{2}}{R^{2}} k \sqrt{2 g} d t
\end{aligned}
$$

Integrando:

$$
\begin{gathered}
\int \frac{1}{\sqrt{h}} d h=-\frac{r^{2}}{R^{2}} k \sqrt{2 g} \int d t \\
2 \sqrt{h}=-\frac{r^{2}}{R^{2}} k \sqrt{2 g} t+c \\
\sqrt{h}=-\frac{r^{2}}{R^{2}} k \frac{\sqrt{2 g}}{2} t+c
\end{gathered}
$$

Para $t=0 \mathrm{~s}$, se sabe que la altura máxima de la celda es $h_{0}=5,943 \mathrm{~cm}$.

$$
\sqrt{5,943}=-\frac{r^{2}}{R^{2}} k \frac{\sqrt{2 g}}{2}(0)+c
$$


Tinoco Robledo

Por lo que tendríamos:

$$
\sqrt{5,943} \mathrm{~cm}=c
$$

Entonces, la altura $h$ en función del tiempo queda:

$$
h(t)=\left[\sqrt{5,943}-\frac{r^{2}}{R^{2}} k \frac{\sqrt{2 g}}{2} t\right]^{2}
$$

Considerando las medidas de los elementos usados en la experimentación tendríamos, para nuestro caso particular, $R=1,35 \mathrm{~cm} \mathrm{y} r=0,9 \mathrm{~cm}$.

Luego:

$$
h(t)=\left[\sqrt{5,943}-\frac{0,9^{2}}{1,35^{2}} k \frac{\sqrt{2 g}}{2} t\right]^{2}
$$

Sabemos que $k$ es un valor que depende de las características del fluido, es decir, tanto del tipo como de la temperatura del mismo. Haciendo un análisis comparativo del modelo expuesto previamente frente al modelo conseguido experimentalmente, se calculó para cada evento el coeficiente de descarga asociado al principio de Torricelli. A continuación, se presentan los datos respectivos de los eventos 1, 2, 3 y 6, empezando desde el modelo teórico a partir de los tiempos dados y terminando tanto en la altura del recipiente de vaciado como en el recipiente de llenado.

\section{Procedimiento}

La altura por considerar en el modelo expuesto previamente corresponde a la altura del fluido en la celda, pero no a la altura de llenado. Al obtener la altura del fluido restante en la celda se calcula también el volumen para cada tiempo; posteriormente, a partir del volumen restante se calcula el volumen del fluido que ha sido vaciado, estableciendo así la altura que logra tal volumen teniendo en cuenta las nuevas condiciones del envase, es decir la probeta que se está llenando. En las tablas presentadas a continuación (tablas 2, 3, 4 y 5), la columna denominada "Altura restante" corresponde al modelo construido desde el principio de Torricelli con una sola variación: el valor del coeficiente de descarga deducido experimentalmente. 
Tabla 2.

Construcción de la altura en la probeta a partir del modelo considerado para el evento 1

\begin{tabular}{|c|c|c|c|c|c|c|}
\hline \multicolumn{7}{|c|}{ Lubricante de transmisión 20 ${ }^{\circ}$ C, coeficiente de descarga $\boldsymbol{k = 0 , 0 1 5 *}$} \\
\hline $\begin{array}{c}\text { Volumen } \\
\text { total }\end{array}$ & Tiempo & $\begin{array}{c}\text { Constante } \\
\sqrt{5,943 \mathrm{~cm}}\end{array}$ & $\begin{array}{c}\text { Altura } \\
\text { restante (cm) }\end{array}$ & $\begin{array}{c}\text { Volumen restante } \\
\text { en la celda Furol }\end{array}$ & $\begin{array}{c}\text { Volumen } \\
\text { desalojado }\end{array}$ & $\begin{array}{c}\text { Altura ganada en } \\
\text { la probeta (cm) }\end{array}$ \\
\hline 34,02 & 0 & 2,4378269 & 5,9430000000 & 34,02 & 0 & 0 \\
\hline 34,02 & 9,2 & 2,4378269 & 5,3086418646 & 30,3949083 & 3,62509171 & 1,2 \\
\hline 34,02 & 18,85 & 2,4378269 & 4,6817173251 & 26,8054189 & 3,58948943 & 2,3 \\
\hline 34,02 & 29,1 & 2,4378269 & 4,0589420766 & 23,2396864 & 3,56573245 & 3,4 \\
\hline 34,02 & 40,4 & 2,4378269 & 3,4238598446 & 19,6034897 & 3,63619673 & 4,4 \\
\hline 34,02 & 52,87 & 2,4378269 & 2,7856954875 & 15,9496461 & 3,6538436 & 5,4 \\
\hline 34,02 & 65,24 & 2,4378269 & 2,2176189409 & 12,6970939 & 3,25255215 & 6,4 \\
\hline 34,02 & 79,45 & 2,4378269 & 1,6449050749 & 9,41799055 & 3,27910337 & 7,4 \\
\hline 34,02 & 94,41 & 2,4378269 & 1,1342336314 & 6,49411433 & 2,92387622 & 8,4 \\
\hline 34,02 & 111,32 & 2,4378269 & 0,6709485338 & 3,84155113 & 2,6525632 & 9,2 \\
\hline 34,02 & 128,39 & 2,4378269 & 0,3259249133 & 1,86610024 & 1,97545089 & 10,2 \\
\hline 34,02 & 147,28 & 2,4378269 & 0,0877447720 & 0,50238731 & 1,36371293 & 10,7 \\
\hline 34,02 & 165,9 & 2,4378269 & 0,0006483456 & 0,00371214 & 0,49867517 & 12,0 \\
\hline
\end{tabular}

Fuente: Elaboración propia.

* Se considera un coeficiente de descarga de 0,015 siendo este el factor calculado experimentalmente que más se acerca al evento real.

Tabla 3.

Construcción de la altura en la probeta a partir del modelo considerado para el evento 2

\begin{tabular}{|c|c|c|c|c|c|c|}
\hline \multicolumn{7}{|c|}{ Miel $30{ }^{\circ} \mathbf{C}$, coeficiente de descarga $\boldsymbol{k}=\mathbf{0 , 0 0 6} *$} \\
\hline $\begin{array}{c}\text { Volumen } \\
\text { total }\end{array}$ & Tiempo & $\begin{array}{c}\text { Constante } \\
\sqrt{5,943 \mathrm{~cm}}\end{array}$ & $\begin{array}{c}\text { Altura } \\
\text { restante (cm) }\end{array}$ & $\begin{array}{c}\text { Volumen restante en } \\
\text { la celda Furol }\end{array}$ & $\begin{array}{c}\text { Volumen } \\
\text { desalojado }\end{array}$ & $\begin{array}{c}\text { Altura ganada en } \\
\text { la probeta (cm) }\end{array}$ \\
\hline 34,02 & 0,00 & 2,4378269 & 5,9430000000 & 34,02 & 0 & 0 \\
\hline 34,02 & 22,50 & 2,4378269 & 5,3220516617 & 30,4716868 & 3,54831321 & 1,1 \\
\hline 34,02 & 49,05 & 2,4378269 & 4,6333897850 & 26,528717 & 3,94296981 & 2,4 \\
\hline 34,02 & 75,48 & 2,4378269 & 3,9952125459 & 22,8747996 & 3,65391736 & 3,5 \\
\hline 34,02 & 102,53 & 2,4378269 & 3,3910056894 & 19,4153815 & 3,45941815 & 4,5 \\
\hline 34,02 & 138,48 & 2,4378269 & 2,6646243058 & 15,2564467 & 4,15893483 & 5,6 \\
\hline
\end{tabular}


Tinoco Robledo

\begin{tabular}{|c|c|c|c|c|c|c|}
\hline 34,02 & 172,68 & 2,4378269 & 2,0547668598 & 11,7646758 & 3,49177089 & 6,7 \\
\hline 34,02 & 209,99 & 2,4378269 & 1,4797136292 & 8,47217823 & 3,29249753 & 7,7 \\
\hline 34,02 & 253,93 & 2,4378269 & 0,9232536236 & 5,2861372 & 3,18604104 & 8,8 \\
\hline 34,02 & 298,86 & 2,4378269 & 0,4893403803 & 2,80174409 & 2,4843931 & 9,6 \\
\hline 34,02 & 336,93 & 2,4378269 & 0,2285769336 & 1,30872926 & 1,49301483 & 10,4 \\
\hline 34,02 & 383,94 & 2,4378269 & 0,0418885134 & 0,23983489 & 1,06889437 & 10,8 \\
\hline 34,02 & 428,84 & 2,4378269 & 0,0031912177 & 0,01827148 & 0,2215634 & 12,0 \\
\hline
\end{tabular}

Fuente: Elaboración propia.

* Se considera un coeficiente de descarga de 0,006 siendo este el factor calculado experimentalmente que más se acerca al evento real.

\section{Tabla 4.}

Construcción de la altura en la probeta a partir del modelo considerado para el evento 3

\begin{tabular}{|c|c|c|c|c|c|c|}
\hline \multicolumn{7}{|c|}{ Lubricante de transmisión 40 ${ }^{\circ} \mathbf{C}$, coeficiente de descarga $\boldsymbol{k = 0 , 0 4 *}$} \\
\hline $\begin{array}{c}\text { Volumen } \\
\text { total }\end{array}$ & Tiempo & $\begin{array}{c}\text { Constante } \\
\sqrt{5,943 \mathrm{~cm}}\end{array}$ & $\begin{array}{c}\text { Altura } \\
\text { restante }(\mathbf{c m})\end{array}$ & $\begin{array}{c}\text { Volumen restante en } \\
\text { la celda Furol }\end{array}$ & $\begin{array}{c}\text { Volumen } \\
\text { desalojado }\end{array}$ & $\begin{array}{c}\text { Altura ganada en } \\
\text { la probeta (cm) }\end{array}$ \\
\hline 34,02 & 0 & 2,4378269 & 5,9430000000 & 34,02 & 0 & 0 \\
\hline 34,02 & 3,3 & 2,4378269 & 5,3354783743 & 30,54856214 & 3,471437862 & 1,1 \\
\hline 34,02 & 6,77 & 2,4378269 & 4,7319824503 & 27,09321447 & 3,455347663 & 2,2 \\
\hline 34,02 & 10,43 & 2,4378269 & 4,1346795587 & 23,67332534 & 3,419889131 & 3,3 \\
\hline 34,02 & 14,54 & 2,4378269 & 3,5119545973 & 20,10788081 & 3,565444529 & 4,4 \\
\hline 34,02 & 19,05 & 2,4378269 & 2,8870778056 & 16,53011587 & 3,577764947 & 5,4 \\
\hline 34,02 & 23,86 & 2,4378269 & 2,2880398281 & 13,10029241 & 3,429823456 & 6,4 \\
\hline 34,02 & 28,61 & 2,4378269 & 1,7647524430 & 10,10418296 & 2,996109454 & 7,3 \\
\hline 34,02 & 34,12 & 2,4378269 & 1,2427411315 & 7,115379731 & 2,988803228 & 8,2 \\
\hline 34,02 & 41,18 & 2,4378269 & 0,7073197435 & 4,049796404 & 3,065583326 & 9,2 \\
\hline 34,02 & 47,87 & 2,4378269 & 0,3382704989 & 1,936785538 & 2,113010866 & 10,2 \\
\hline 34,02 & 54,17 & 2,4378269 & 0,1137849249 & 0,651481574 & 1,285303965 & 10,6 \\
\hline 34,02 & 62,05 & 2,4378269 & 0,0010089367 & 0,00577672 & 0,645704853 & 12 \\
\hline
\end{tabular}

Fuente: Elaboración propia.

* Se considera un coeficiente de descarga de 0,04 siendo este el factor calculado experimentalmente que más se acerca al evento real. 
Tabla 5.

Construcción de la altura en la probeta a partir del modelo considerado para el evento 6

\begin{tabular}{|c|c|c|c|c|c|c|}
\hline \multicolumn{7}{|c|}{ Miel 60 ${ }^{\circ}$ C, coeficiente de descarga $\boldsymbol{k}=\mathbf{0 , 0 1 1}$} \\
\hline $\begin{array}{c}\text { Volumen } \\
\text { total }\end{array}$ & Tiempo & $\begin{array}{c}\text { Constante } \\
\sqrt{5,943 \mathrm{~cm}}\end{array}$ & $\begin{array}{c}\text { Altura } \\
\text { restante (cm) }\end{array}$ & $\begin{array}{c}\text { Volumen restante } \\
\text { en la celda Furol }\end{array}$ & $\begin{array}{c}\text { Volumen } \\
\text { desalojado }\end{array}$ & $\begin{array}{c}\text { Altura ganada en } \\
\text { la probeta (cm) }\end{array}$ \\
\hline 34,02 & 0 & 2,4378269 & 5,9430000000 & 34,02 & 0 & 0 \\
\hline 34,02 & 13,15 & 2,4378269 & 5,2789771202 & 30,2250612 & 3,79493877 & 1,2 \\
\hline 34,02 & 27,18 & 2,4378269 & 4,6138790965 & 26,4170075 & 3,80805373 & 2,4 \\
\hline 34,02 & 41,83 & 2,4378269 & 3,9671660258 & 22,7142178 & 3,70278971 & 3,3 \\
\hline 34,02 & 57,15 & 2,4378269 & 3,3430848340 & 19,1410081 & 3,5732097 & 4,4 \\
\hline 34,02 & 73,29 & 2,4378269 & 2,7433373356 & 15,7071222 & 3,43388586 & 5,4 \\
\hline 34,02 & 91,81 & 2,4378269 & 2,1281415761 & 12,1847866 & 3,52233569 & 6,2 \\
\hline 34,02 & 111,66 & 2,4378269 & 1,5553720283 & 8,90536438 & 3,27942218 & 7,3 \\
\hline 34,02 & 132,11 & 2,4378269 & 1,0590013326 & 6,06336785 & 2,84199653 & 8,1 \\
\hline 34,02 & 155,11 & 2,4378269 & 0,6143713572 & 3,51761553 & 2,54575232 & 9,3 \\
\hline 34,02 & 180,67 & 2,4378269 & 0,2613873449 & 1,496587 & 2,02102853 & 10,4 \\
\hline 34,02 & 208,4 & 2,4378269 & 0,0464673841 & 0,26605145 & 1,23053554 & 10,7 \\
\hline 34,02 & 236,66 & 2,4378269 & 0,0073592862 & 0,04213598 & 0,22391547 & 12 \\
\hline
\end{tabular}

Fuente: Elaboración propia.

* Se considera un coeficiente de descarga de 0,011 siendo este el factor calculado experimentalmente que más se acerca al evento real.

\section{CONCLUSIONES}

De acuerdo con las experimentaciones trabajadas y sus mismas variaciones se destacan las siguientes conclusiones, teniendo en cuenta las características principales y los parámetros definidos para cada uno de los eventos.

Tipo de fluido. Se trabajó con dos fluidos: miel y lubricante de transmisión. Comparativamente, hablando de los eventos en los que se expusieron los fluidos a la misma temperatura bajo el mismo tipo de columna, siempre tardó menos tiempo en alcanzar la altura deseada el lubricante de transmisión. Lo enunciado se puede evidenciar desde la comparación de los eventos 4 y 7, donde bajo las mismas condiciones el lubricante tardó 33 s en vaciarse, mientras que la miel tardó 216,82 s. La comparación también puede establecerse entre los eventos 5 y 8, donde la miel tardó aproximadamente el triple de tiempo que tardó el lubricante en vaciarse. 
Temperatura. Según los eventos considerados, y teniendo en cuenta aquellos que cuentan con condiciones similares, se evidencia que a una mayor temperatura se logra una mayor velocidad en el vaciado. Lo expuesto se puede evidenciar desde la comparación entre los eventos 1 y 3, donde se trabajó con lubricante a $40{ }^{\circ} \mathrm{C}$ y $60{ }^{\circ} \mathrm{C}$ respectivamente; en el evento 1 se alcanzó la altura deseada en 166 s aproximadamente, mientras que en el evento 3 se logró este objetivo en $65 \mathrm{~s}$. La afirmación también puede corroborarse a partir de la comparación de los eventos 2 y 6 , los eventos 4 y 5 , y los eventos 7 y 8.

Tipo de columna. Según los eventos considerados, se plantearon en el proceso de investigación dos tipos de columna: la columna variable el caso en el que se perdía altura del fluido en la celda Furol conforme avanzaba el tiempo, y la columna constante el caso en el que la altura fuese siempre la misma a partir del llenado constante de la celda que se encontraba vaciándose. En el primer caso se encontró para cada uno de los eventos (1, 2, 3 y 6) que la velocidad de llenado en la probeta no era constante, sino que disminuía conforme la altura de la celda de vaciado era menor, afirmación que puede corroborarse a partir de los modelos expuestos previamente, donde se evidencia que la altura está definida por una función cuadrática cuya velocidad de llenado se define a partir de un modelo lineal decreciente.

En el segundo caso se encuentran los eventos 4, 5, 7 y 8. En todos ellos se trabajó con columna constante, evidenciando que bajo las condiciones descritas la velocidad de llenado en la probeta permaneció constante, lo que originó modelos lineales crecientes para relacionar la altura con el tiempo transcurrido.

En conclusión, comparativamente hablando, en los eventos bajo las mismas condiciones de temperatura y tipo de fluido, pero con tipos de columna distinta, fue evidente que, al tener una velocidad constante dada por columnas constantes, el tiempo de llenado de la probeta en la que se depositó el fluido siempre fue menor (eventos $3 \mathrm{y}$ 4, y eventos 6 y 8 ).

Dado que la columna del fluido resultó ser un elemento para destacar dentro de las hipótesis propuestas inicialmente, se presenta en el siguiente apartado la justificación teórica de lo evidenciado experimentalmente, que se relaciona estrictamente con el principio de Torricelli.

Temperatura ascendente con columna variable. Fue un único evento en el que se varió la temperatura de manera que esta aumentara conforme transcurría el tiempo mientras la altura del fluido disminuía. Teniendo en cuenta las variables descritas, al aumentar la temperatura se esperaría una mayor velocidad en el llenado de la probeta, mientras que con la disminución de la altura de la columna de fluido se espera que la velocidad disminuya. Finalmente, desde el análisis de la experimentación el modelo que mejor se ajustó fue una función cuadrática con una velocidad que es directamente proporcional al tiempo trascurrido, lo que significa que a mayor tiempo mayor velocidad de caudal. Esto se puede evidenciar directamente en la tabla del evento 9, donde se utilizó una probeta con una altura de $24 \mathrm{~cm}$, y para el llenado de los primeros $12 \mathrm{~cm}$ transcurrieron $677,38 \mathrm{~s}$, mientras que para los siguientes $12 \mathrm{~cm}$ transcurrieron apenas 
260 s. Aquí es evidente que el aumento de la temperatura vence la disminución de la velocidad de llenado debida a la pérdida de altura de fluido.

En relación con el pensamiento covariacional. El proceso desarrollado alrededor de la modelación y la simulación del llenado de una botella y sus variaciones aquí descrito es el resultado de la primera parte de un proyecto de investigación con fines educativos, asociado a la formación en el ciclo básico de ingeniería. A partir de los resultados obtenidos, se determina hasta aquí que el desarrollo del pensamiento covariacional se da en el investigador, en la medida que le permite pasar de la experimentación a la simulación, de la realidad a la aproximación teórica, a partir de la integración de conocimientos previos y de la observación. Además, dentro de los experimentos modelados en particular, el pensamiento covariacional le permite al investigador interpretar la relación entre las variables asociadas al problema, identificando las correlaciones en la altura del fluido y la razón de cambio de esta con respecto al tiempo (velocidad de llenado) dado el tipo de columna, la temperatura y las características del fluido.

Aquí se hace evidente entonces la necesidad de fortalecer el pensamiento covariacional entre los estudiantes de carreras afines a la ingeniería. Esto hace necesario dar continuidad a la investigación bajo un enfoque de aplicación, con un proceso inverso al aquí evidenciado, reemplazando la experimentación por la simulación propuesta.

El investigador presentará entonces a los estudiantes una situación problema en la que ellos deberán esbozar las gráficas que relacionan algunas de las variables presentadas aquí (altura en función del tiempo, velocidad de llenado en función del tiempo, volumen en función del tiempo), y a partir de allí les corresponderá a ellos obtener tanta información como les sea necesaria para representar correctamente dicha situación sin ningún proceso de experimentación, lo que se traduce en la búsqueda de variables y de los cambios simultáneos de estas. Posterior a ello las hipótesis podrán ser contrastadas con la simulación, de lo cual se deben desprender nuevos resultados, con la expectativa de que estos sean no solo más cercanos a la realidad, sino mejor justificados desde los conocimientos adquiridos en los primeros cursos de matemáticas y física.

\section{Referencias}

Acofi (2007). El ingeniero colombiano del 2020: Retos para su formación. Bogotá: Acofi.

Carlson, M. P., Jacobs, S., Coe, E., Larsen, S., \& Hsu, E. (2002). Applying covariational reasoning while modeling dynamic events: A framework and a study. Journal for Research in Mathematics Education, 33(5), 352-378.

Clement, J. (1989). The concept of variation and misconceptions in Cartesian graphing. Focus on Learning Problems in Mathematics, 11(1-2), 77-87.

Confrey, J., \& Smith, E. (1994). Exponential functions, rates of change, and the multiplicative unit. Educational Studies in Mathematics, 26, 135-164. 
Jhonson, H. L. (2012). Reasoning about variation in the intensity of change in covarying quantities involved in rate of change. Journal of Mathematical Behavior, 31, 313-330.

Johnson, H. L. (2013). Reasoning about quantities that change together. The Mathematics Teacher, 106(9), 704-708.

Johnson, H. L. (2015). Together yet separate: Students' associating amounts of change in quantities involved in rate of change. Educational Studies in Mathematics, 89, 89-110.

Moore, K. C., \& Thompson, P. W. (2015). Shape thinking and students' graphing activity. En T. Fukawa-Connelly, N. Infante, K. Keene \& M. Zandieh (Eds.), Proceedings of the eighteenth annual conference on research in undergraduate mathematics education (pp. 782-789). Pittsburgh: Mathematical Association of America.

Paoletti, T., \& Moore, K. C. (2017). The parametric nature of two students' covariational reasoning. Journal of Mathematical Behaviour, 48, 137-151.

Saldanha, L., \& Thompson, P. W. (1998). Re-thinking covariation from a quantitative perspective: Simultaneous continuous variation. En S. B. Berenson, K. R. Dawkins, M. Blanton, W. N. Coloumbe, J. Kolb, K. Norwood, \& L. Stiff (eds.), Proceedings of the 20th annual meeting of the psychology of mathematics education North American chapter (pp. 298-303). Raleigh, NC: North Carolina State University.

Stalvey, H. \& Vidakovic, D. (2015). Students' reasoning about relationships between variables in a real-world problem. Journal of Mathematical Behaviour, 40, 192-210.

Simon, M. A. (1996). Beyond inductive and deductive reasoning: The search for a sense of knowing. Educational Studies in Mathematics, 30(2), 197-209.

Thompson P. W. (2011). Quantitative reasoning and mathematical modeling. En New perspectives and directions for collaborative research in mathematics education, (Vol. 1). 